sac pressure and diameter over time. An ultrasound-activated remote pressure transducer was implanted into 21 patients who underwent endovascular aneurysm repair of an infrarenal AAA. At regular follow-up visits, sac pressure was measured by remote sensor and compared with the patient's systemic arterial pressure. Aneurysms were defined as shrinking if the maximum sac diameter decreased by $\geq 5 \mathrm{~mm}$.

Among 14 patients followed up for at least 6 months for whom sac pressure measurements could be obtained, 7 individuals had shrinking aneurysms and marked reductions in sac pressure. As only one transducer could be implanted in each patient, the authors point out that the sac pressure measurements might have been affected by compartmentalization. Modifications of the deployment technique are planned to improve the reliability of long-term pressure monitoring.

Claire Braybrook

Original article Ellozy SH et al. (2006) Abdominal aortic aneurysm sac shrinkage after endovascular aneurysm repair: correlation with chronic sac pressure measurement. J Vasc Surg 43: 2-7

\section{Dyslipidemia prevalence, treatment and control in a multiethnic population}

Treating dyslipidemia can lower the risk of coronary heart disease. The National Cholesterol Education Program's third report of the Adult Treatment Panel (ATP III) makes recommendations to health-care professionals regarding the diagnosis and treatment of dyslipidemia. The Multi-Ethnic Study of Atherosclerosis (MESA) investigated the prevalence and treatment of dyslipidemia in a cohort of healthy individuals to assess the challenges facing implementation of these guidelines.

In this study, 6,814 individuals without clinical cardiovascular disease (mean age 62.0 years) completed an initial physical examination. The prevalence of dyslipidemia, as defined by ATP III, was $29.3 \%$ overall. Dyslipidemia prevalence was similar among non-Hispanic white, black and Hispanic participants, but less prevalent in Chinese individuals. Overall, $54.0 \%$ of patients with dyslipidemia were receiving lipid-lowering treatment. Dyslipidemia was more prevalent in older age-groups than in younger age-groups and approximately $30 \%$ more common in men than in women. Men with dyslipidemia were about $20 \%$ less likely to receive treatment than women with the condition, and $30 \%$ less likely to have their dyslipidemia controlled. Control of dyslipidemia was less common in individuals at high and intermediate risk of cardiovascular disease than in low-risk individuals.

Dyslipidemia, as defined by ATP III, is common in individuals without known clinical cardiovascular disease, but treatment and control are suboptimal, particularly in those at higher risk of cardiovascular disease. Further research is needed to improve the treatment and control of this condition, and to eliminate any disparities between different patient groups.

Rebecca Ireland

Original article Goff DC Jr et al. (2006) Dyslipidemia prevalence, treatment, and control in the Multi-Ethnic Study of Atherosclerosis (MESA): gender, ethnicity, and coronary artery calcium. Circulation 113: $647-656$

\section{Percutaneous retrograde transarterial aortic valve implantation successful}

Percutaneous retrograde transarterial aortic valve implantation via the femoral artery is successful in selected high-risk patients with symptomatic aortic stenosis, according to a recent study published in Circulation. This technique offers an alternative to the antegrade transvenous approach, which has been associated with technical difficulties and high risk.

Webb et al. attempted percutaneous valve implantation in 18 patients (aged 75-87 years) who were poor candidates for surgery because of comorbidities. The equipment and techniques were designed specifically for a transarterial approach to prosthetic valve implantation, including a Cribier-Edwards ${ }^{\circledR}$ valve (Edwards Lifesciences, Irvine, CA), a 22F/24F sheath, which was advanced from the femoral artery to the aorta, and a deflectable guiding catheter and rapid right ventricular pacing to facilitate deployment of the prosthesis. Results were consequently better than those achieved by some previous studies, with implantation being successful in 14 of 18 patients. Aortic valve area following implantation increased from $0.6 \pm 0.2 \mathrm{~cm}^{2}$ to $1.6 \pm 0.4 \mathrm{~cm}^{2}$, and no deaths occurred during the procedure. Two patients died during $73 \pm 49$ days' follow-up; one because of iliac perforation and the other most probably from left coronary 